PROCEEDINGS OF THE

AMERICAN MATHEMATICAL SOCIETY

Volume 130, Number 6, Pages 1699-1710

S 0002-9939(01)06239-6

Article electronically published on December 20, 2001

\title{
ESSENTIAL SPECTRUM OF A SYSTEM OF SINGULAR DIFFERENTIAL OPERATORS AND THE ASYMPTOTIC HAIN-LÜST OPERATOR
}

\author{
REINHARD MENNICKEN, SERGUEI NABOKO, AND CHRISTIANE TRETTER
}

(Communicated by Joseph A. Ball)

\begin{abstract}
We consider a matrix differential operator with singular entries which arises in magnetohydrodynamics. By means of the asymptotic HainLüst operator and some pseudo-differential operator techniques, we determine the essential spectrum of this operator. Whereas in the regular case, the essential spectrum consists of two intervals, it turns out that in the singular case two additional intervals due to the singularity may arise. In addition, we establish criteria for the essential spectrum to lie in the left half-plane.
\end{abstract}

\section{INTRODUCTION}

The spectral analysis of systems of matrix differential operators of mixed order (ordinary and partial) is a fast developing part of mathematical physics having various applications in different fields (plasma physics, hydrodynamics, astrophysics, etc.). Recently, problems where the coefficients of the equations have prescribed singularities attracted the attention of specialists working in operator theory and applications. These problems appeared in a natural way in magnetohydrodynamics and led to some new phenomena due to the singularities (see e.g. [HMN]). In the present paper we use a special problem of this kind in order to apply a new approach to the spectral analysis of matrix differential operators with singularities, which might allow a better understanding of other concrete problems and of the general case.

In [HMN] self-adjoint $3 \times 3$ block operator matrices the entries of which are singular differential operators of different orders have been studied. Block operator matrices of this kind are related to a simple physical model describing the oscillations of a plasma in an equilibrium configuration confined in a cylindrical domain

Received by the editors May 3, 2000 and, in revised form, December 5, 2000.

1991 Mathematics Subject Classification. Primary 47A10, 47B25, 76W05.

The authors acknowledge the support of the Regensburger Universitätsstiftung Hans Vielberth.

(C)2001 American Mathematical Society 
(see [K2]). They are of the form

$$
\left(\begin{array}{c|cc}
\partial_{r} \frac{\Gamma}{r} \partial_{r} r+q & \mathrm{i} \partial_{r} \frac{\beta_{1}}{r}+\gamma_{1} & \mathrm{i} \partial_{r} \beta_{2} \\
\hline \frac{\beta_{1}}{r^{2}} \mathrm{i} \partial_{r} r+\bar{\gamma}_{1} & \frac{d_{11}}{r^{2}} & \frac{d_{12}}{r} \\
\frac{\beta_{2}}{r} \mathrm{i} \partial_{r} r & \frac{d_{12}}{r} & d_{22}
\end{array}\right)
$$

where $\Gamma, \beta_{1}, \beta_{2}, \gamma_{1}, q$ and $d_{i j}$ are sufficiently smooth functions on the interval [0, $\left.r_{0}\right]$, $r_{0}$ denoting the radius of the cylinder. The crucial difficulty in studying such block operator matrices is that the two terms $A D$ and $B C$ in the formal determinant $A D-B C$ (see (2.1) below) have the same order 2 as differential operators as well as the same order 4 of the singularity at $r=0$.

The main results of [HMN] are the construction of a self-adjoint extension $\mathbb{L}$ of the operator in (1.1) in the Hilbert space $\left(L_{2}\left(0, r_{0}\right), r \mathrm{~d} r\right)^{3}$ (along the lines of [K1]) if the coefficients $\Gamma, \beta_{1}, \beta_{2}, d_{11}, d_{12}$ in (1.1) fulfill so-called quasi-regularity conditions, and a description of the essential spectrum of this self-adjoint extension provided that $\Re\left(\gamma_{1}(0)\right)=0$ and $d_{12}+\beta_{1} \beta_{2} / \Gamma \equiv 0$. Compared with the so-called hard core problem (see [K1, [DG]), where the operator in (1.1) is considered in $\left(L_{2}\left(r_{1}, r_{0}\right), r \mathrm{~d} r\right)^{3}$ with some $0<r_{1}<r_{0}$ and is thus regular, it turned out that in the singular case additional components of essential spectrum may appear, which was predicted in [DG]. However, the restriction $d_{12}+\beta_{1} \beta_{2} / \Gamma \equiv 0$ led to only one new interval of essential spectrum.

The aim of the present paper is not only to extend the description of the essential spectrum to the general case where no assumptions on $\Re\left(\gamma_{1}(0)\right)$ and $d_{12}+\beta_{1} \beta_{2} / \Gamma$ are imposed, but merely to develop a new and much more elegant way to determine this essential spectrum and to avoid tiresome calculations. The main tool here is an abstract lemma which allows us to reduce this problem to the problem of determining the essential spectrum of a matrix function. The part of the essential spectrum due to the singularity (singularity spectrum for short 1 ) predicted in [DG] consists of two intervals determined by the roots of the so-called asymptotic HainLüst operator. We also study the location of the various parts of the essential spectrum, and we address the question of stability of the above system of differential operators by deriving a criterion for the essential spectrum of $\mathbb{L}$ to lie in the left half-plane.

In our opinion the definition and use of asymptotic Hain-Lüst operators is of crucial importance and will turn out to be a proper tool for the investigation of the new branches of the essential spectra for a wide class of systems of matrix differential operators with singularities. It should be mentioned that another approach to the study of the essential spectrum of matrix differential operators has been developed in [FMM1], FMM2]. Also, we would like to add that the results of the present paper may be extended to the case when $\mathbb{L}$ is non-self-adjoint since there exists a non-self-adjoint analogue of the above mentioned lemma.

\footnotetext{
${ }^{1}$ It should be noted that there is no relation with the singular spectrum of the operator.
} 


\section{Preliminaries}

If we substitute $r=r(t)=r_{0} \mathrm{e}^{-t}$, the operator in (1.1) takes the form

$$
\mathbb{L}_{0}^{\prime}=\left(\begin{array}{c|cc}
\partial_{t} \frac{\Gamma}{r^{2}} \partial_{t}+q & \mathrm{i} \partial_{t} \frac{\beta_{1}}{r^{2}}+\gamma_{1} & \mathrm{i} \partial_{t} \frac{\beta_{2}}{r} \\
\hline \frac{\beta_{1}}{r^{2}} \mathrm{i} \partial_{t}+\bar{\gamma}_{1} & \frac{d_{11}}{r^{2}} & \frac{d_{12}}{r} \\
\frac{\beta_{2}}{r} \mathrm{i} \partial_{t} & \frac{d_{12}}{r} & d_{22}
\end{array}\right)=:\left(\begin{array}{cc}
A & B \\
C & D
\end{array}\right) .
$$

Here and in the sequel we use both variables $r$ and $t$ simultaneously for convenience. We consider this operator in the Hilbert space $H=\left(L_{2}\left(\mathbb{R}_{+}\right)\right)^{3}$ on the domain

$$
\mathcal{D}\left(\mathbb{L}_{0}^{\prime}\right):=\left[H_{2, \mathrm{fin}}\left(\mathbb{R}_{+}\right) \cap \stackrel{\circ}{H}_{1}\left(\mathbb{R}_{+}\right)\right] \oplus H_{1, \mathrm{fin}}\left(\mathbb{R}_{+}\right) \oplus H_{1, \mathrm{fin}}\left(\mathbb{R}_{+}\right) .
$$

Here, for $k \in \mathbb{N}_{0}, H_{k}\left(\mathbb{R}_{+}\right)$denotes the Sobolev space of order $k$ associated with $L_{2}\left(\mathbb{R}_{+}\right), H_{k, \text { fin }}\left(\mathbb{R}_{+}\right)$is the space of all functions $f$ in $H_{k}\left(\mathbb{R}_{+}\right)$with support in some finite interval $[0, c]$, and $\stackrel{\circ}{H} k\left(\mathbb{R}_{+}\right)$stands for the closure of $C_{0}^{\infty}\left(\mathbb{R}_{+}\right)$with respect to the norm in $H_{k}\left(\mathbb{R}_{+}\right)$.

In this paper we will always assume that the following conditions are satisfied:

a) The functions $\Gamma, \beta_{1}, \beta_{2}, d_{11}, d_{12}, d_{22}$ have continuous derivatives up to order 3 on $\left[0, r_{0}\right], \Gamma>0$ on $\left[0, r_{0}\right]$, the functions $q, \gamma_{1}$ have continuous derivatives (as functions of $r$ ) up to order 2 on $\left[0, r_{0}\right]$, and $q, \beta_{1}, \beta_{2}, d_{11}, d_{22}$ are real-valued.

b) The functions $d_{11}, d_{12}, d_{22}$ satisfy the conditions

$$
\begin{gathered}
d_{11}(r) \leq 0, \quad d_{22}(r) \leq 0, \quad d_{11}(r) d_{22}(r) \geq\left|d_{12}(r)\right|^{2}, \quad r \in\left[0, r_{0}\right], \\
d_{11}(0)<0 .
\end{gathered}
$$

In [HMN] it has been shown that $\mathbb{L}_{0}^{\prime}$ is symmetric. The following theorem from [HMN] provides conditions which guarantee that $\mathbb{L}_{0}^{\prime}$ has a self-adjoint extension.

Theorem 2.1. Let the assumptions a) and $\mathrm{b}$ ) hold and suppose that the coefficients $\Gamma, \beta_{1}, \beta_{2}, d_{11}$ and $d_{12}$ satisfy the so-called quasi-regularity conditions

$$
\frac{1}{r^{2}}\left(d_{11}+\frac{\beta_{1}^{2}}{\Gamma}\right)=\mathrm{O}(1), \quad \frac{1}{r}\left(d_{12}+\frac{\beta_{1} \beta_{2}}{\Gamma}\right)=\mathrm{O}(1) \quad(r \searrow 0) .
$$

Then the operator $\mathbb{L}_{0}^{\prime}$ has a self-adjoint extension $\mathbb{L}$ which is given by

$$
\mathcal{D}(\mathbb{L})=R\left(M_{\mu}\right), \quad \mathbb{L}:=M_{\mu}^{-1}+\mu I,
$$

where $\mu$ is an arbitrary sufficiently large positive number and

$$
M_{\mu}:=\left(\begin{array}{cc}
T^{-1}(\mu) & -F(\mu)^{*} \\
-F(\mu) & (D-\mu I)^{-1}+(D-\mu I)^{-1} C F(\mu)^{*}
\end{array}\right) .
$$

Here $T(\mu)$ with domain $H_{2}\left(\mathbb{R}_{+}\right) \cap \stackrel{\circ}{H}_{1}\left(\mathbb{R}_{+}\right)$is the so-called Hain-Lüst operator, i.e., the (self-adjoint) closure of the transfer function

$$
T_{0}(\mu):=A-\mu I-B(D-\mu I)^{-1} C
$$

defined on $\mathcal{D}(A)=H_{2, \mathrm{fin}}\left(\mathbb{R}_{+}\right) \cap \stackrel{\circ}{H}_{1}\left(\mathbb{R}_{+}\right)$and $F(\mu)$ is the bounded operator on $L_{2}\left(\mathbb{R}_{+}\right)$given by

$$
F(\mu):=(D-\mu I)^{-1} C T^{-1}(\mu)
$$


Note that all products of operators occurring in (2.7) - (2.9) are well-defined according to $[\mathrm{HMN}$, and that the theorem on the pseudo-resolvent (see e.g. $[\mathrm{Y}]$ ), by means of which the self-adjoint extension $\mathbb{L}$ has been constructed in [HMN] (and in $[\mathrm{K} 2$ for a special case), guarantees that the domain of $\mathbb{L}$ is independent of $\mu$.

From the representation of $\mathbb{L}$ in Theorem 2.1 it readily follows that the essential spectrum of $\mathbb{L}$ is determined by the essential spectrum of the operator $M_{\mu}$ via

$$
\lambda \in \sigma_{\mathrm{ess}}(\mathbb{L}) \Longleftrightarrow \frac{1}{\lambda-\mu} \in \sigma_{\mathrm{ess}}\left(M_{\mu}\right) .
$$

In order to determine its essential spectrum, the operator $M_{\mu}$ has been reduced in $[\mathrm{HMN}$ modulo compact operators. The next theorem is a consequence of this reduction. To formulate it, we need the following definitions and relations. We set

$$
Q_{2}(\cdot, \mu):=r^{2} \operatorname{det}(D-\mu I)=r^{2}\left(\mu^{2}-\mu d_{22}\right)+d_{11}\left(d_{22}-\mu\right)-\left|d_{12}\right|^{2},
$$

and with

$$
a:=\frac{\Gamma}{r^{2}}, \quad b:=\left(\frac{\beta_{1}}{r^{2}}, \frac{\beta_{2}}{r}\right)
$$

we define

$$
A(\cdot, \mu):=a+b(D-\mu I)^{-1} b^{*} .
$$

Further, we introduce

$$
S_{0}:=\left(\begin{array}{cc}
s_{11} & s_{12} \\
\frac{s_{12}}{} & s_{22}
\end{array}\right):=\left(\begin{array}{cc}
\frac{1}{r^{2}}\left(d_{11}+\frac{\beta_{1}^{2}}{\Gamma}\right) & \frac{1}{r}\left(d_{12}+\frac{\beta_{1} \beta_{2}}{\Gamma}\right) \\
\frac{1}{r}\left(\overline{d_{12}}+\frac{\beta_{1} \beta_{2}}{\Gamma}\right) & d_{22}+\frac{\beta_{2}^{2}}{\Gamma}
\end{array}\right)
$$

and

$$
P_{2}(\cdot, \mu):=\Gamma \operatorname{det}\left(S_{0}-\mu I\right) .
$$

In the following the values of (operator) functions at the point $r=0$ are to be understood as limits when $r \searrow 0$ (observe that $r \searrow 0$ corresponds to $t \rightarrow \infty$ ).

It has been shown in [HMN, Lemma 5.4] that if conditions a), b) and the quasiregularity conditions (2.5) are satisfied, then, for $\mu>0, A(\cdot, \mu)$ is continuously differentiable in $\left[0, r_{0}\right]$ and has a limit as $r \searrow 0$,

$$
A(\mu):=\lim _{r \searrow 0} A(r, \mu)=\frac{\Gamma(0)}{Q_{2}(0, \mu)} \operatorname{det}\left(\begin{array}{cc}
\frac{1}{2} f_{1}^{\prime \prime}(0)-\mu & f_{2}^{\prime}(0) \\
\overline{f_{2}^{\prime}(0)} & d_{22}(0)+\frac{\beta_{2}^{2}(0)}{\Gamma(0)}-\mu
\end{array}\right)
$$

where

$$
f_{1}:=d_{11}+\frac{\beta_{1}^{2}}{\Gamma}, \quad f_{2}:=d_{12}+\frac{\beta_{1} \beta_{2}}{\Gamma} .
$$

Finally, we define the so-called asymptotic Hain-Lüst operator $T_{\text {as }}(\mu)$ in $L_{2}\left(\mathbb{R}_{+}\right)$ by replacing all coefficients in $T_{0}(\mu)$ by their limits for $r \searrow 0$, i.e.,

$$
\begin{aligned}
& \mathcal{D}\left(T_{\mathrm{as}}(\mu)\right):=\mathcal{D}(T(\mu)):=H_{2}\left(\mathbb{R}_{+}\right) \cap \stackrel{\circ}{H}_{1}\left(\mathbb{R}_{+}\right), \\
& T_{\text {as }}(\mu)=\partial_{t} A(\mu) \partial_{t}-2 \Re\left(\gamma_{1}(0)\right) \frac{\beta_{1}(0)}{d_{11}(0)} \mathrm{i} \partial_{t}+q(0)-\mu,
\end{aligned}
$$


and we introduce the $2 \times 2$ matrix functions

$$
\begin{aligned}
& \widetilde{F}_{1}(\cdot, \mu):=-A(\cdot, \mu)^{-1}(D-\mu I)^{-1} b^{*} b(D-\mu I)^{-1}, \\
& \widehat{F}_{1}(\cdot, \mu):=(D-\mu I)^{-1}+\widetilde{F}_{1}(\cdot, \mu) .
\end{aligned}
$$

In the following we denote equality of linear operators modulo compact operators (i.e., equality in the Calkin algebra) by $\stackrel{\mathrm{C}}{=}$.

Theorem 2.2. Suppose that assumptions a) and b) and the quasi-regularity conditions (2.5) are satisfied. Then, for all sufficiently large positive numbers $\mu$,

$$
\widehat{F}_{1}(0, \mu)=\frac{1}{Q_{2}(0, \mu)}\left(\left(\begin{array}{cc}
0 & 0 \\
0 & d_{11}(0)
\end{array}\right)-\frac{\beta_{1}(0)^{2}}{P_{2}(0, \mu)}\left(\begin{array}{cc}
g(\mu)^{2} & -f_{2}^{\prime}(0) g(\mu) \\
-\overline{f_{2}^{\prime}(0)} g(\mu) & \left|f_{2}^{\prime}(0)\right|^{2}
\end{array}\right)\right)
$$

where

$$
g(\mu):=s_{22}(0)-\mu=d_{22}(0)+\frac{\beta_{2}(0)^{2}}{\Gamma(0)}-\mu
$$

and for the operator $M_{\mu}$ we have a representation

$$
M_{\mu} \stackrel{\mathrm{C}}{=} \widetilde{M}_{\mu}=Q_{\mu}+X_{\mu}+P_{\mu}
$$

with a constant matrix $Q_{\mu}$, a matrix multiplication operator $X_{\mu}$ in the variable $t$, and a matrix pseudo-differential operator $P_{\mu}$ with a symbol constant in $t$, given by

$$
\begin{aligned}
& Q_{\mu}:=\left(\begin{array}{cc}
0 & 0 \\
0 & \widehat{F}_{1}(0, \mu)
\end{array}\right), \quad X_{\mu}:=\left(\begin{array}{cc}
0 & 0 \\
0 & \widehat{F}_{1}(\cdot, \mu)-\widehat{F}_{1}(0, \mu)
\end{array}\right), \\
& P_{\mu}:=\left(\begin{array}{c|c}
T_{\mathrm{as}}^{-1}(\mu) & \beta(\mu)\left(\mathrm{i} \partial_{t} T_{\mathrm{as}}^{-1}(\mu)\right)^{*} \\
\hline \overline{\beta(\mu)}^{t} \mathrm{i} \partial_{t} T_{\mathrm{as}}^{-1}(\mu) & -\widetilde{F}_{1}(0, \mu)+\widetilde{F}_{1}(0, \mu) \partial_{t} A(\mu) \partial_{t} T_{\mathrm{as}}^{-1}(\mu)
\end{array}\right)
\end{aligned}
$$

where

$$
\beta(\mu):=\left(-\frac{\beta_{1}(0)}{d_{11}(0)}, \frac{f_{2}^{\prime}(0) \beta_{1}(0)}{Q_{2}(0, \mu)}\right) .
$$

Proof. In [HMN, (4.6)] it has been shown that

$$
A(\cdot, \mu)=\frac{P_{2}(\cdot, \mu)}{Q_{2}(\cdot, \mu)} .
$$

This, the fact that

$$
\begin{aligned}
\lim _{r \searrow 0} \frac{1}{r}\left(-\beta_{1} d_{12}+\beta_{2} d_{11}\right) & =\lim _{r \searrow 0}-\beta_{1} \frac{1}{r}\left(d_{12}+\frac{\beta_{1} \beta_{2}}{\Gamma}\right)+\beta_{2} r \frac{1}{r^{2}}\left(d_{11}+\frac{\beta_{1}^{2}}{\Gamma}\right) \\
& =-\beta_{1}(0) f_{2}^{\prime}(0)
\end{aligned}
$$

due to the quasi-regularity conditions (2.5), and

$$
\lim _{r \searrow 0}(D-\mu I)^{-1}(r)=\lim _{r \searrow 0} \frac{r^{2}}{Q_{2}(\cdot, \mu)}\left(\begin{array}{cc}
d_{22}-\mu & -\frac{d_{12}}{r} \\
-\frac{d_{12}}{r} & \frac{d_{11}}{r^{2}}-\mu
\end{array}\right)=\left(\begin{array}{cc}
0 & 0 \\
0 & \frac{d_{11}(0)}{Q_{2}(0, \mu)}
\end{array}\right)
$$


by the definition of $D$ in (2.2) and of $Q_{2}(\cdot, \mu)$ in (2.11) yield the representation of $\widehat{F}_{1}(0, \mu)$ according to (2.18). The representation of $M_{\mu}$ follows from [HMN] Theorem 6.9], observing that

$$
-2 \Re\left(\gamma_{1}(0)\right) \frac{\beta_{1}(0)}{d_{11}(0)} \mathrm{i} \partial_{t}+q(0)-\mu=T_{\text {as }}(\mu)-\partial_{t} A(\mu) \partial_{t}
$$

in the right lower corner of $P_{\mu}$.

\section{Reduction to matrix CASE}

In order to be able to apply the Fourier transformation, we extend the operator $\widetilde{M}_{\mu}$ in Theorem 2.2 to an operator $\widetilde{M}_{\mu, \mathbb{R}}$ on $\mathbb{R}$ such that $\sigma\left(\widetilde{M}_{\mu}\right)=\sigma\left(\widetilde{M}_{\mu, \mathbb{R}}\right)$ (by considering the differentiation on $\mathbb{R}$ and replacing $r(t)=r_{0} \mathrm{e}^{-t}$ by $r(|t|)$ in the coefficients). The operator $\widetilde{M}_{\mu, \mathbb{R}}$ is (up to a finite rank operator) equal to

$$
\widetilde{M}_{\mu, \mathbb{R}} \stackrel{\mathrm{C}}{=} \widehat{M}_{\mu, \mathbb{R}}=Q_{\mu, \mathbb{R}}+X_{\mu, \mathbb{R}}+P_{\mu, \mathbb{R}}
$$

where $Q_{\mu, \mathbb{R}}, X_{\mu, \mathbb{R}}$ arise from the operators $Q_{\mu}, X_{\mu}$ in Theorem 2.2 by replacing $r(t)$ by $r(|t|)$, and $P_{\mu, \mathbb{R}}$ arises from the operator $P_{\mu}$ in Theorem 2.2 by replacing $T_{\text {as }}^{-1}(\mu)$ by the operator $T_{\mathrm{as}, \mathbb{R}}^{-1}(\mu)$ given by

$$
\begin{aligned}
& \mathcal{D}\left(T_{\mathrm{as}, \mathbb{R}}(\mu)\right):=H_{2}(\mathbb{R}), \\
& T_{\mathrm{as}, \mathbb{R}}(\mu):=\partial_{t} A(\mu) \partial_{t}-2 \Re\left(\gamma_{1}(0)\right) \frac{\beta_{1}(0)}{d_{11}(0)} \mathrm{i} \partial_{t}+q(0)-\mu .
\end{aligned}
$$

For details we refer the reader to the paper [HMN].

By what we have shown so far, the essential spectrum of $M_{\mu}$ coincides with the essential spectrum of $\widehat{M}_{\mu, \mathbb{R}}$,

$$
\sigma_{\text {ess }}\left(M_{\mu}\right)=\sigma_{\text {ess }}\left(\widehat{M}_{\mu, \mathbb{R}}\right) .
$$

By means of the Fourier transformation and the following abstract lemma, the calculation of the essential spectrum of the operator $\widehat{M}_{\mu, \mathbb{R}}$ (and consequently of $\mathbb{L}$ due to (2.10) and (3.2) ) is reduced to the calculation of the essential spectra of matrix functions.

Lemma 3.1. Let $\mathcal{M}$ be a pseudo-differential operator in $L_{2}(\mathbb{R})^{n}$ with matrix symbol $M(x, p)$ having a representation

$$
M(x, p)=Q+X(x)+P(p)
$$

where the variable $p$ corresponds to $\mathrm{i} \partial_{x}$ and

i) $Q$ is a constant Hermitian $n \times n$ matrix,

ii) $X$ and $P$ are (multiplication operators with) hermitian $n \times n$ matrix functions with entries from $L_{\infty}(\mathbb{R})$ such that $\lim _{|x| \rightarrow \infty} X(x)=0, \lim _{|p| \rightarrow \infty} P(p)=0$.

Then

$$
\sigma_{\text {ess }}(\mathcal{M}) \backslash \sigma(Q)=\left(\sigma_{\text {ess }}(Q+X) \cup \sigma_{\text {ess }}(Q+P)\right) \backslash \sigma(Q) .
$$

Proof. This lemma is a special case of a result proved in [KN].

In the sequel we will show that this lemma applies to the operator $\widehat{M}_{\mu, \mathbb{R}}$, and we will calculate the respective two parts of the essential spectrum due to $Q_{\mu, \mathbb{R}}+X_{\mu, \mathbb{R}}$ (the regular part) and to $Q_{\mu, \mathbb{R}}+P_{\mu, \mathbb{R}}$ (the new part due to the singularity at $r=0$ ). 


\section{Calculation of the Regular part of the essential spectrum}

In this section we calculate the essential spectrum of the operator

$$
Q_{\mu, \mathbb{R}}+X_{\mu, \mathbb{R}}=\left(\begin{array}{cc}
0 & 0 \\
0 & \widehat{F}_{1}(\cdot, \mu)
\end{array}\right)
$$

By [HW, Proposition 2.3 and Example 2.3], we obtain

$\sigma_{\mathrm{ess}}\left(Q_{\mu, \mathbb{R}}+X_{\mu, \mathbb{R}}\right)=\{0\} \cup \overline{\left\{\sigma \in \mathbb{R}: \operatorname{det}\left(\widehat{F}_{1}(t, \mu)-\sigma I\right)=0 \text { for some } t \in[0, \infty)\right\}}$.

By the definition of $\widehat{F}_{1}(\cdot, \mu)$ in (2.18), the condition $\operatorname{det}\left(\widehat{F}_{1}(\cdot, \mu)-\sigma I\right)=0$ is equivalent to

$$
\operatorname{det}\left((D-\mu I)^{-1}-A(\cdot, \mu)^{-1}(D-\mu I)^{-1} b^{*} b(D-\mu I)^{-1}-\sigma\right)=0 .
$$

Since $\operatorname{det}(D-\mu I)^{-1} \neq 0$, this is further equivalent to

$$
\operatorname{det}\left(D-\frac{1+\sigma \mu}{\sigma} I+\frac{1}{\sigma} A(\cdot, \mu)^{-1}(D-\mu I)^{-1} b^{*} b\right)=0
$$

for $\sigma \neq 0$. If we set $\lambda:=\frac{1+\sigma \mu}{\sigma}$, then $\sigma=\frac{1}{\lambda-\mu}$. Observing that $A(\cdot, \mu)$ is scalar, using the Hilbert identity $(\lambda-\mu)(D-\lambda I)^{-1}(D-\mu I)^{-1}=(D-\lambda I)^{-1}-(D-\mu I)^{-1}$ and twice [K] Chap. III, Problem 4.17], we can rewrite the right-hand side of [4.1] as

$$
\begin{aligned}
& \operatorname{det}(D-\lambda I) \operatorname{det}\left(I+A(\cdot, \mu)^{-1}\left((D-\lambda I)^{-1}-(D-\mu I)^{-1}\right) b^{*} b\right) \\
& =\operatorname{det}(D-\lambda I) \operatorname{det}\left(1+A(\cdot, \mu)^{-1} b\left((D-\lambda I)^{-1}-(D-\mu I)^{-1}\right) b^{*}\right) \\
& =A(\cdot, \lambda) A(\cdot, \mu)^{-1} \operatorname{det}(D-\lambda I) \\
& =a\left(1+a^{-1} b(D-\lambda I)^{-1} b^{*}\right) A(\cdot, \mu)^{-1} \operatorname{det}(D-\lambda I) \\
& =a\left(I+a^{-1}(D-\lambda I)^{-1} b^{*} b\right) A(\cdot, \mu)^{-1} \operatorname{det}(D-\lambda I) \\
& =a A(\cdot, \mu)^{-1} \operatorname{det}\left(S_{0}-\lambda I\right)
\end{aligned}
$$

with $S_{0}$ given by (2.14) where we have used (2.11), (2.12). So, for $\mu$ sufficiently large,

$$
\operatorname{det}\left(\widehat{F}_{1}(\cdot, \mu)-\sigma I\right)=0 \quad \Longleftrightarrow \quad \operatorname{det}\left(S_{0}-\lambda I\right)=0
$$

According to assumptions a), b) and the quasi-regularity conditions (2.5), all entries of the symmetric matrix function $S_{0}$ depend continuously on $t$ and have a limit when $t \rightarrow \infty$. Hence there exist two real-valued continuous functions $\omega_{1}, \omega_{2}$, the ranges of which are finite intervals such that

$$
\operatorname{det}\left(S_{0}(t)-\lambda\right)=\left(\lambda-\omega_{1}(t)\right)\left(\lambda-\omega_{2}(t)\right) .
$$

Consequently,

$$
\sigma_{\mathrm{ess}}\left(Q_{\mu, \mathbb{R}}+X_{\mu, \mathbb{R}}\right)=\{0\} \cup \overline{\left\{\sigma \in \mathbb{R}: \frac{1}{\sigma}+\mu \in \omega_{1}([0, \infty)) \cup \omega_{2}([0, \infty))\right\}}
$$

Note that if we set $\sigma=\frac{1}{\lambda-\mu}$ in view of (2.10), the description of $\sigma_{\mathrm{ess}}\left(Q_{\mu, \mathbb{R}}+X_{\mu, \mathbb{R}}\right)$ depends only on $\lambda$ as it should be according to the resolvent-like construction of $M_{\mu}(\operatorname{see}(\underline{2.6})$. 


\section{Calculation of the singularity part OF THE ESSENTIAL SPECTRUM}

Now we determine the essential spectrum of the operator $Q_{\mu, \mathbb{R}}+P_{\mu, \mathbb{R}}$. Since $Q_{\mu, \mathbb{R}}+P_{\mu, \mathbb{R}}$ is a matrix pseudo-differential operator with constant coefficients, by means of the Fourier transformation, it is unitarily equivalent to the operator of multiplication with its symbol. Therefore its essential spectrum is given by

$$
\sigma_{\text {ess }}\left(Q_{\mu, \mathbb{R}}+P_{\mu, \mathbb{R}}\right)=\overline{\left\{\sigma \in \mathbb{R}: \operatorname{det}\left(Q_{\mu, \mathbb{R}}+P_{\mu, \mathbb{R}}(p)-\sigma I\right)=0 \text { for some } p \in \mathbb{R}\right\}} \text {. }
$$

According to the representation of $Q_{\mu}, P_{\mu}$ and hence of $Q_{\mu, \mathbb{R}}, P_{\mu, \mathbb{R}}$ according to Theorem 2.2, the condition

$$
\operatorname{det}\left(Q_{\mu, \mathbb{R}}+P_{\mu, \mathbb{R}}(p)-\sigma I\right)=0
$$

is equivalent to

$\operatorname{det}\left(\left(\begin{array}{cc}0 & 0 \\ 0 & \widehat{F}_{1}(0, \mu)-\widetilde{F}_{1}(0, \mu)\end{array}\right)+T_{\text {as }, \mathbb{R}}^{-1}(\mu)(p)\left(\begin{array}{cc}1 & \beta(\mu) p \\ \frac{\beta(\mu)}{t}^{t} p & -\widetilde{F}_{1}(0, \mu) A(\mu) p^{2}\end{array}\right)-\sigma I\right)=0$.

Using the fact that due to (2.5)

$-\widetilde{F}_{1}(0, \mu) A(\mu)=\frac{\beta_{1}(0)^{2}}{Q_{2}(0, \mu)^{2}}\left(\begin{array}{cc}g(\mu)^{2} & -f_{2}^{\prime}(0) g(\mu) \\ -\overline{f_{2}^{\prime}(0)} g(\mu) & \left|f_{2}^{\prime}(0)\right|^{2}\end{array}\right),\left|d_{12}(0)\right|^{2}=-d_{11}(0) \frac{\beta_{2}(0)^{2}}{\Gamma(0)}$

and thus $Q_{2}(0, \mu)=d_{11}(0) g(\mu)=d_{11}(0)\left(s_{22}(0)-\mu\right)$, it is easy to see that

$$
-\widetilde{F}_{1}(0, \mu) A(\mu)=\overline{\beta(\mu)}^{t} \beta(\mu) .
$$

Therefore and because of (2.19) the condition (5.1) is equivalent to

$$
\operatorname{det}\left(\left(\begin{array}{lll}
0 & 0 & 0 \\
0 & 0 & 0 \\
0 & 0 & 1
\end{array}\right) \frac{1}{s_{22}(0)-\mu}+T_{\mathrm{as}, \mathbb{R}}^{-1}(\mu)(p)\left(\begin{array}{cc}
1 & \beta(\mu) p \\
\overline{\beta(\mu)}^{t} p & \frac{\beta}{\beta(\mu)} t^{t} \beta(\mu) p^{2}
\end{array}\right)-\sigma I\right)=0 .
$$

Let $\beta(\mu)=:\left(b_{1}, b_{2}\right)$. If we define the unitary $3 \times 3$ matrix $U$ by

$$
U:=\left(\begin{array}{cc}
V & 0 \\
0 & 1
\end{array}\right), \quad V:=\frac{1}{\sqrt{1+b_{1}^{2} p^{2}}}\left(\begin{array}{cc}
b_{1} p & 1 \\
-1 & b_{1} p
\end{array}\right)
$$

and multiply the matrix in the above determinant by $U^{*}$ from the left and by $U$ from the right, we obtain

$$
\left(\begin{array}{ccc}
0 & 0 & 0 \\
0 & 0 & 0 \\
0 & 0 & 1
\end{array}\right) \frac{1}{s_{22}(0)-\mu}+T_{\mathrm{as}, \mathbb{R}}^{-1}(\mu)(p)\left(\begin{array}{ccc}
0 & 0 & 0 \\
0 & 1+b_{1}^{2} p^{2} & b_{2} p \sqrt{1+b_{1}^{2} p^{2}} \\
0 & \frac{b_{2}}{p} \sqrt{1+b_{1}^{2} p^{2}} & \left|b_{2}\right|^{2} p^{2}
\end{array}\right)-\sigma I .
$$

Hence, if $\sigma \neq 0$, the condition (5.1) is equivalent to

$$
\operatorname{det}\left(\begin{array}{cc}
1+b_{1}^{2} p^{2}-\sigma T_{\mathrm{as}, \mathbb{R}}(\mu)(p) & b_{2} p \sqrt{1+b_{1}^{2} p^{2}} \\
\overline{b_{2}} p \sqrt{1+b_{1}^{2} p^{2}} & \left(\frac{1}{s_{22}(0)-\mu}-\sigma\right) T_{\mathrm{as}, \mathbb{R}}(\mu)(p)+\left|b_{2}\right|^{2} p^{2}
\end{array}\right)=0
$$

which simplifies to

$$
T_{\mathrm{as}, \mathbb{R}}(\mu)(p)\left(\left(\frac{1}{s_{22}(0)-\mu}-\sigma\right)\left(1+b_{1}^{2} p^{2}-\sigma T_{\mathrm{as}, \mathbb{R}}(\mu)(p)\right)-\sigma\left|b_{2}\right|^{2} p^{2}\right)=0 .
$$


Since for $\mu \gg 0$ the operator $T_{\mathrm{as}, \mathbb{R}}(\mu)$ is invertible, the above relation is equivalent to

$$
\left(\frac{1}{s_{22}(0)-\mu}-\sigma\right) T_{\mathrm{as}, \mathbb{R}}(\mu)(p)=\left(\frac{1}{\sigma\left(s_{22}(0)-\mu\right)}-1\right)\left(1+b_{1}^{2} p^{2}\right)-\left|b_{2}\right|^{2} p^{2} .
$$

By the definition of $T_{\text {as }, \mathbb{R}}(\mu)$ which is induced by the formula in (2.17) and by (2.16) we conclude straightforwardly

$$
T_{\text {as }, \mathbb{R}}(\mu)(p)=T_{\text {as }, \mathbb{R}}(\lambda)(p)+(\lambda-\mu)\left(1+b_{1}^{2} p^{2}\right)-b_{1}^{2} p^{2}\left(\frac{\left|f_{2}^{\prime}(0)\right|^{2}}{s_{22}(0)-\mu}-\frac{\left|f_{2}^{\prime}(0)\right|^{2}}{s_{22}(0)-\lambda}\right) .
$$

After some calculations one finds that this implies, for $\sigma=\frac{1}{\lambda-\mu}$,

$$
\left(\sigma-\frac{1}{s_{22}(0)-\mu}\right)\left(T_{\mathrm{as}, \mathbb{R}}(\mu)(p)-T_{\mathrm{as}, \mathbb{R}}(\lambda)(p)\right)=1+\left(b_{1}^{2}+\left|b_{2}\right|^{2}\right) p^{2}-\frac{1+b_{1}^{2} p^{2}}{\sigma\left(s_{22}(0)-\mu\right)}
$$

Using this last relation, we see that (5.2) and hence (5.1) is equivalent to

$$
\left(\frac{1}{\lambda-\mu}-\frac{1}{s_{22}(0)-\mu}\right) T_{\mathrm{as}, \mathbb{R}}(\lambda)(p)=0 \text {. }
$$

Thus we have shown

$$
=\frac{\sigma_{\mathrm{ess}}\left(Q_{\mu, \mathbb{R}}+P_{\mu, \mathbb{R}}\right) \backslash\{0\}}{\left\{\sigma \in \mathbb{R}:\left(\frac{1}{\lambda-\mu}-\frac{1}{s_{22}(0)-\mu}\right) T_{\mathrm{as}, \mathbb{R}}(\lambda)(p)=0 \text { for some } p \in \mathbb{R}\right\}}
$$

where $\lambda=\frac{1}{\sigma}-\mu$, i.e., $\sigma=\frac{1}{\lambda-\mu}$. It should be observed that only in the special case $f_{2}^{\prime}(0)=0$ the point $\lambda=s_{22}(0)$ appears as a zero of the above equation because otherwise, if $f_{2}^{\prime}(0) \neq 0, T_{\text {as, } \mathbb{R}}(\lambda)$ has a singularity at $\lambda=s_{22}(0)$ (see (2.17), (2.16) and hence nominator and denominator in (5.3) cancel each other.

\section{The Essential SPECTRUM of $\mathbb{L}$, LOCATION OF ITS PARTS AND STABility}

The following theorem describing the essential spectrum of $\mathbb{L}$ is our main result.

Theorem 6.1. Let $\omega_{1}(t), \omega_{2}(t), t \in[0, \infty)$, be the roots of the quadratic equation $\operatorname{det}\left(S_{0}(t)-\lambda\right)=0$ with $S_{0}$ given by (2.14), and let $\lambda_{1}(p), \lambda_{2}(p), p \in \mathbb{R}$, be the roots of the quadratic equation (in the variable $\lambda$ )

$$
\left(s_{22}(0)-\lambda\right) T_{\mathrm{as}, \mathbb{R}}(\lambda)(p)=0
$$

where $T_{\mathrm{as}, \mathbb{R}}$ is the asymptotic Hain-Lüst operator defined in (2.17). Then the essential spectrum of the self-adjoint extension $\mathbb{L}$ of the operator $\mathbb{L}_{0}^{\prime}$ in (2.1) is given by

$$
\sigma_{\text {ess }}(\mathbb{L})=\sigma_{\text {ess,reg }}(\mathbb{L}) \cup \sigma_{\text {ess,sing }}(\mathbb{L})
$$

where

$$
\begin{aligned}
\sigma_{\text {ess,reg }}(\mathbb{L}) & =\overline{\omega_{1}([0, \infty))} \cup \overline{\omega_{2}([0, \infty))}, \\
\sigma_{\text {ess,sing }}(\mathbb{L}) & =\overline{\lambda_{1}((-\infty, \infty))} \cup \overline{\lambda_{2}((-\infty, \infty))} .
\end{aligned}
$$

Proof. The assertion follows from Lemma 3.1, (4.2) and (5.3) if we observe the relation (2.10) between the essential spectrum of $\mathbb{L}$ and that of $M_{\mu}$. Further, one has to note that the point $\sigma=0$ corresponds to $\lambda=\infty$, that for different values of $\mu$ the sets of the two other points of the spectrum of $Q_{\mu, \mathbb{R}}$ have empty intersection, and that in the case $f_{2}^{\prime}(0)=0$ the point $s_{22}(0)$ already belongs to $\sigma_{\text {ess,reg }}(\mathbb{L})$. 
The next proposition shows that the two intervals $\overline{\lambda_{1}((-\infty, \infty))}, \overline{\lambda_{2}((-\infty, \infty))}$ of the singularity spectrum are adjoined to the two intervals $\overline{\omega_{1}([0, \infty))}, \overline{\omega_{2}([0, \infty))}$ of the regular spectrum, and it provides a criterion when the singularity spectrum disappears, i.e., collapses into two points belonging to the regular part of the spectrum. Note that even if regular and singularity spectrum overlap, the latter does not disappear due to its multiplicity.

Proposition 6.2. The pairs of intervals $\overline{\omega_{1}([0, \infty))}, \overline{\omega_{2}([0, \infty))}$ and $\overline{\lambda_{1}((-\infty, \infty))}$, $\overline{\lambda_{2}((-\infty, \infty))}$ can be enumerated such that

$$
\omega_{1}(0) \in \overline{\lambda_{1}((-\infty, \infty))}, \quad \omega_{2}(0) \in \overline{\lambda_{2}((-\infty, \infty))} .
$$

The two intervals $\overline{\lambda_{1}((-\infty, \infty))}, \overline{\lambda_{2}((-\infty, \infty))}$ collapse into the two points $\omega_{1}(0)$, $\omega_{2}(0)$ disappearing in the regular part of the spectrum if and only if

$$
f_{2}^{\prime}(0)=0, \quad \Re\left(\gamma_{1}(0)\right)=0, \quad q(0)=\frac{f_{1}^{\prime \prime}(0)}{2} .
$$

Proof. Due to the quasi-regularity conditions (2.5) and the definition of $S_{0}$ in (2.14), the points $\omega_{1}(0), \omega_{2}(0)$ are the zeros of the quadratic equation

$$
\left(\frac{1}{2} f_{1}^{\prime \prime}(0)-\lambda\right)\left(s_{22}(0)-\lambda\right)-\left|f_{2}^{\prime}(0)\right|^{2}=0 .
$$

On the other hand, observing the definition of $A(\cdot)$ in (2.16) and the facts that according to the quasi-regularity conditions (2.5)

$$
\left|d_{12}(0)\right|^{2}=-d_{11}(0) \frac{\beta_{2}(0)^{2}}{\Gamma(0)}, \quad \frac{\beta_{1}(0)}{d_{11}(0)}=-\frac{\Gamma(0)}{\beta_{1}(0)},
$$

we see that the equation (6.1) is equivalent to

$$
\begin{aligned}
& \left(\left(\frac{1}{2} f_{1}^{\prime \prime}(0)-\lambda\right)\left(s_{22}(0)-\lambda\right)-\left|f_{2}^{\prime}(0)\right|^{2}\right) \frac{\Gamma(0)^{2}}{\beta_{1}(0)^{2}} p^{2} \\
& +2 \Re\left(\gamma_{1}(0)\right) \frac{\Gamma(0)}{\beta_{1}(0)}\left(s_{22}(0)-\lambda\right) p+(q(0)-\lambda)\left(s_{22}(0)-\lambda\right)=0 .
\end{aligned}
$$

In the limit $p \rightarrow \pm \infty$ this equation is equivalent to (6.3), which proves the first assertion. If we set $\widetilde{p}:=\frac{\Gamma(0)}{\beta_{1}(0)} p$ and $\widetilde{\lambda}:=\lambda-s_{22}(0)$, the equation (6.1) reads

$\widetilde{\lambda}^{2}\left(\widetilde{p}^{2}+1\right)+\widetilde{\lambda}\left(\left(-\frac{f_{1}^{\prime \prime}(0)}{2}+s_{22}(0)\right) \widetilde{p}^{2}-2 \Re\left(\gamma_{1}(0)\right) \widetilde{p}-q(0)+s_{22}(0)\right)-\left|f_{2}^{\prime}(0)\right|^{2} \widetilde{p}^{2}=0$.

The solutions of this equation coincide for all $p \in \mathbb{R}$ and hence $\widetilde{p} \in \mathbb{R}$ if and only if

$$
\frac{\left(-\frac{f_{1}^{\prime \prime}(0)}{2}+s_{22}(0)\right) \widetilde{p}^{2}-2 \Re\left(\gamma_{1}(0)\right) \widetilde{p}-q(0)+s_{22}(0)}{\widetilde{p}^{2}+1} \text { and } \frac{-\left|f_{2}^{\prime}(0)\right|^{2} \widetilde{p}^{2}}{\widetilde{p}^{2}+1}
$$

are constant for all $\widetilde{p} \in \mathbb{R}$ which is equivalent to the conditions (6.2).

Theorem 6.1 also enables us to formulate a criterion for the essential spectrum of $\mathbb{L}$ to be located in the left half-plane, which is connected with the 'stability' of the system of differential operators (1.1). 
Theorem 6.3. The essential spectrum of $\mathbb{L}$ is contained in the half-line $\mathbb{R}_{-}:=$ $\{z \in \mathbb{R}: z \leq 0\}$ if and only if

$$
\left(d_{11}+\frac{\beta_{1}^{2}}{\Gamma}\right)\left(d_{22}+\frac{\beta_{2}^{2}}{\Gamma}\right) \geq\left|d_{12}+\frac{\beta_{1} \beta_{2}}{\Gamma}\right|^{2}, \quad d_{11}+\frac{\beta_{1}^{2}}{\Gamma} \leq 0, \quad d_{22}+\frac{\beta_{2}^{2}}{\Gamma} \leq 0,
$$

and one of the following 4 conditions is satisfied:

i) $s_{22}(0)=0, f_{1}^{\prime \prime}(0)=0, \Re\left(\gamma_{1}(0)\right)=0, f_{2}^{\prime}(0)=0, q(0) \leq 0$,

ii) $s_{22}(0)=0, f_{1}^{\prime \prime}(0)<0, f_{2}^{\prime}(0)=0, q(0) \leq \frac{\Re\left(\gamma_{1}(0)\right)^{2}}{\frac{f_{1}^{\prime \prime}(0)}{2}}$,

iii) $s_{22}(0)<0, \frac{f_{1}^{\prime \prime}(0)}{2} s_{22}(0)=\left|f_{2}^{\prime}(0)\right|^{2}, \Re\left(\gamma_{1}(0)\right)=0, q(0) \leq 0$,

iv) $s_{22}(0)<0, \frac{f_{1}^{\prime \prime}(0)}{2} s_{22}(0)>\left|f_{2}^{\prime}(0)\right|^{2}, q(0) \leq \frac{\Re\left(\gamma_{1}(0)\right)^{2}}{\frac{f_{1}^{\prime \prime}(0)}{2}-\frac{\left|f_{2}^{\prime}(0)\right|^{2}}{s_{22}(0)}}$,

where $s_{22}=d_{22}+\beta_{2}^{2} / \Gamma$.

Proof. The conditions (6.5) are equivalent to the fact that all zeros $\omega_{1}(t), \omega_{2}(t)$ of the quadratic equation $\operatorname{det}\left(S_{0}(t)-\lambda\right)=0$ (and hence $\left.\sigma_{\text {ess,reg }}(\mathbb{L})\right)$ lie in $\mathbb{C}_{-}$. Further, all zeros $\lambda_{1}(p), \lambda_{2}(p)$ of the quadratic equation (6.1) (and hence $\sigma_{\text {ess,sing }}(\mathbb{L})$ ) lie in $\mathbb{C}_{-}$if and only if in the equation (6.4) the coefficient of $\lambda$ and the constant coefficient both are nonnegative, i.e.,

$$
\begin{aligned}
& -\left(\frac{f_{1}^{\prime \prime}(0)}{2}+s_{22}(0)\right) \widetilde{p}^{2}-2 \Re\left(\gamma_{1}(0)\right) \widetilde{p}-q(0)-s_{22}(0) \geq 0, \\
& \left(\frac{f_{1}^{\prime \prime}(0)}{2} s_{22}(0)-\left|f_{2}^{\prime}(0)\right|^{2}\right) \widetilde{p}^{2}+2 \Re\left(\gamma_{1}(0)\right) s_{22}(0) \widetilde{p}+q(0) s_{22}(0) \geq 0
\end{aligned}
$$

for all $\widetilde{p} \in \mathbb{R}$ where we have set $\widetilde{p}:=\frac{\Gamma(0)}{\beta_{1}(0)} p$. It is not difficult to see that this is the case if and only if one of the conditions i) to iv) in Theorem 6.3 holds.

\section{REFERENCES}

[DG] Descloux, J., Geymonat, G., Sur le spectre essentiel d'un operateur relatif à la stabilité d'un plasma en géometrie toroïdale, C. R. Acad. Sci. Paris Sér. A-B 290 (1980), 795-797. MR 81e:76087

[FMM1] Faierman, M., Mennicken, R., Möller, M., The essential spectrum of a system of singular ordinary differential operators of mixed order. Part I: The general problem and an almost regular case, Math. Nachr. 208 (1999), 101-115. MR 2000i:34156

[FMM2] Faierman, M., Mennicken, R., Möller, M., The essential spectrum of a system of singular ordinary differential operators of mixed order. Part II: The generalization of Kako's problem, Math. Nachr. 209 (2000), 55-81. MR 2000i:34155

[HMN] Hardt, V., Mennicken, R., Naboko, S.N., Systems of singular differential operators of mixed order and applications to 1-dimensional MHD problems, Math. Nachr. 205 (1999), 19-68. MR 2000f:47073

[HW] Hardt, V., Wagenführer, E., Spectral properties of a multiplication operator, Math. Nachr. 178 (1996), 135-156. MR 97i:47058

[K1] Kako, T., On the absolutely continuous spectrum of MHD plasma confined in the flat torus, Math. Methods Appl. Sci. 7 (1985), 432-442. MR 87d:76159

[K2] Kako, T., Essential spectrum of linearized operator for MHD plasma in cylindrical region, Z. Angew. Math. Phys. 38 (1987), 433-449. MR 89c:76117

[K] Kato, T., Perturbation Theory for Linear Operators, Springer-Verlag, Berlin, Heidelberg, 1995. MR 96a:47025 
[KN] Kurasov, P., Naboko, S.N., On the spectrum of a class of singular matrix-differential operators. Quasiregularity conditions and essential self-adjointness, to be submitted to Trans. Amer. Math. Soc.

[Y] Yoshida, K., Functional Analysis, Springer-Verlag, Berlin, Heidelberg, New York, 1968.

NWF I - Mathematik, University of Regensburg, D-93040 Regensburg, Germany

E-mail address: reinhard.mennicken@mathematik.uni-regensburg.de

Department of Mathematical Physics, Institute for Physics, St. Petersburg UniverSity, ul. Ulianovskaja 1, 198904 St. Petergoff, St. Petersburg, Russia

E-mail address: naboko@snoopy.phys.spbu.ru

Department of Mathematics and Computer Science, University of Leicester, UniverSity RoAd, Leicester LE1 7RH, United Kingdom

E-mail address: c.tretter@mcs.le.ac.uk 